\title{
THE NEW HORIZON RUN COSMOLOGICAL $N$-BODY SIMULATIONS
}

\author{
Juhan Kim ${ }^{1}$, Changbom Park ${ }^{2}$, Graziano Rossi ${ }^{2}$, Sang Min LeE ${ }^{3}$, and J. Richard Gott III $^{4}$ \\ ${ }^{1}$ Center for Advanced Computation, Korea Institute for Advanced Study, 85 Hoegiro, Dongdaemun-gu, Seoul \\ 130-722, Korea \\ E-mail : kjhan@kias.re.kr \\ 2 School of Physics, Korea Institute for Advanced Study, 85 Hoegiro, Dongdaemun-gu, Seoul 130-722, Korea \\ ${ }^{3}$ Supercomputing Center, KISTI, 335 Gwahangno, Yuseong-gu, Daejon, 305-806 Korea \\ ${ }^{4}$ Department of Astrophysical Sciences, Princeton University, Princeton, NJ 08550, USA \\ (Received November 9, 2011; Revised November 22, 2011; Accepted December 2, 2011)
}

\begin{abstract}
We present two large cosmological $N$-body simulations, called Horizon Run 2 (HR2) and Horizon Run 3 (HR3), made using $6000^{3}=216$ billions and $7210^{3}=374$ billion particles, spanning a volume of $\left(7.200 h^{-1} \mathrm{Gpc}\right)^{3}$ and $\left(10.815 h^{-1} \mathrm{Gpc}\right)^{3}$, respectively. These simulations improve on our previous Horizon Run 1 (HR1) up to a factor of 4.4 in volume, and range from 2600 to over 8800 times the volume of the Millennium Run. In addition, they achieve a considerably finer mass resolution, down to $1.25 \times 10^{11} h^{-1} \mathrm{M}_{\odot}$, allowing to resolve galaxy-size halos with mean particle separations of $1.2 h^{-1} \mathrm{Mpc}$ and $1.5 h^{-1} \mathrm{Mpc}$, respectively. We have measured the power spectrum, correlation function, mass function and basic halo properties with percent level accuracy, and verified that they correctly reproduce the $\Lambda \mathrm{CDM}$ theoretical expectations, in excellent agreement with linear perturbation theory. Our unprecedentedly large-volume $N$-body simulations can be used for a variety of studies in cosmology and astrophysics, ranging from large-scale structure topology, baryon acoustic oscillations, dark energy and the characterization of the expansion history of the Universe, till galaxy formation science - in connection with the new SDSS-III. To this end, we made a total of 35 all-sky mock surveys along the past light cone out to $z=0.7$ ( 8 from the HR2 and 27 from the HR3), to simulate the BOSS geometry. The simulations and mock surveys are already publicly available at http://astro.kias.re.kr/Horizon-Run23/.
\end{abstract}

Key words : cosmological parameters - cosmology: theory - large-scale structure of the Universe galaxies: formation - methods: $N$-body simulations

\section{INTRODUCTION}

State-of-the-art observations from the Cosmic Microwave Background (CMB), such as data provided by the Wilkinson Microwave Anisotropy Probe (WMAP; Spergel et al. 2003, Komatsu et al. 2011), and from the Large Scale Structure (LSS) as in the Sloan Digital Sky Survey (SDSS; York et al. 2000), in the 2dF galaxy redshift survey (Colless et al. 2001) and in the WiggleZ survey (Blake et al. 2008), support a model of the Universe dominated by Cold Dark Matter (CDM) and Dark Energy (DE), with baryons constituting only a percent of the total matter-energy content. Structures form and grow hierarchically, from the smallest objects to the largest ones. In this framework, dark matter collapses first into small haloes which accrete matter and merge to form progressively larger halos over time. Galaxies form subsequently, out of gas which cools and collapses to the centers of dark matter halos (White \& Rees 1978; Peebles 1982; Davis et al. 1985); therefore, halo and galaxy properties are strongly related. In synergy with independent data from Type I super-

Corresponding Author: G. Rossi (graziano@kias.re.kr) novae (SN Ia; Riess et al. 1998, Perlmutter et al. 1999, Kowalski et al. 2008), we also have increasing confirmations that the Universe is geometrically flat, and currently undergoing a phase of accelerated expansion.

What is driving this expansion, and in particular the nature of dark energy, still remains to be explained. To date, it is perhaps one of the most important open questions in cosmology, which would deeply impact our understanding of fundamental physics (see for example Albrecht et al. 2006). In addition, investigating the nature of the (nearly) Gaussian primordial fluctuations - which eventually led to the formation of halos and galaxies - is also of utmost importance for shedding light into the structure formation process. In fact, constraining possible deviations from Gaussianity will impact for example the LSS topology (Gott et al. 1986; Park et al. 1998, 2005; Gott et al. 2009; Choi et al. 2010), as any primordial non-Gaussianity might modify the clustering properties of massive cosmic structures forming out of rare density fluctuations (LoVerde et al. 2008; Desjacques et al. 2009; Jeong \& Komatsu 2009), and generate a scale-dependent large-scale bias in the clustering properties of massive dark matter halos (Dalal et al. 2008; Verde \& Matarrese 2009; Des- 
jacques \& Seljak 2010; Jee et al. 2011). Moreover, studying global halo properties such as halo formation, density profiles, concentrations, shapes, kinematics, assembly times, spin, velocity distributions, substructures and the effects of baryons is particularly important in order to gain insights into the formation and evolution of galaxies, their large-scale environment in the cosmic web (Bett et al. 2007; Gao \& White 2007; Jing Suto \& Mo 2007; Li et al. 2008; Gott et al. 2009; Shandarin et al. 2010), and to test the validity of cosmological models.

Therefore, at the moment there is considerable interest in constraining cosmological models (i.e., the main cosmological parameters) from real datasets with percent level accuracy, and especially the dark energy equation of state (DE EoS). In particular, most of the efforts towards characterizing DE and its properties are focused on measurements of the baryon acoustic oscillation (BAO) scale. The BAO distinct signature, imprinted in the large-scale galaxy distribution by acoustic fluctuations in the baryon-photon fluid prior to recombination, appears as a quasi-harmonic series of oscillations of decreasing amplitude in the galaxy power spectrum at wavenumbers $0.01 \mathrm{hMpc}^{-1} \leq k \leq$ $0.4 \mathrm{Mpc}^{-1}$ (Sugiyama 1995; Eisenstein \& $\mathrm{Hu} 1998$, 1999), or as a broad and quasi-Gaussian peak in the corresponding two-point correlation function (Matsubara 2004). The measurement of its scale is often achieved by using a well-controlled sample of luminous red galaxies (LRGs), as observed for example in the SDSS or in the 2dFGRS surveys (Colless et al. 2001; Eisenstein et al. 2005; Cole et al. 2005; Sanchez et al. 2006, 2009; Percival et al. 2007, 2010; Gaztanaga et al. 2009; Kazin et al. 2010; Reid et al. 2010; Carnero et al. 2011). Since the BAO scale depends solely on the plasma physics after the Big Bang and can be calibrated using CMB data, it can be used as a standard ruler to measure the redshift dependence of the Hubble parameter $H(z)$ and the angular diameter distance (Montesano et al. 2010), and thus to constrain the DE EoS parameter $w(z)$ as a function of redshift - along with the other main cosmological parameters. Complementary to the BAO technique, the LSS topology can also be used as a standard ruler, by measuring and comparing the amplitude of the genus curve at different redshifts and smoothing lengths (see Park \& Kim 2010). Cosmological parameters can also be obtained through the abundance of high-mass halos, identified as galaxy clusters, and via several other methods that require and utilize precise knowledge of galaxy clustering (i.e., Zheng \& Weinberg 2007; Yoo et al. 2009).

To this end, planned next generation photometric redshift galaxy surveys will span volumes considerably larger than the current data sets, providing a dramatic improvement in the accuracy of the constraints on cosmological parameters. Examples are the Panoramic Survey Telescope \& Rapid Response System (Pan-STARRS; Kaiser et al. 2002), the Dark Energy Survey (DES; Abbott et al. 2005), the Baryonic
Oscillation Spectroscopic Survey (BOSS; Schlegel et al. 2009) as well as BigBOSS (Schlegel et al. 2011), the Large Synoptic Survey Telescope (LSST; Tyson et al. 2004), the Hobby Eberly Telescope Dark Energy Experiment (HETDEX; Hill et al. 2004), and the space-based Wide-Field Infrared Survey Telescope (WFIRST; Green et al. 2011) and Euclid (Cimatti et al. 2008). The lack of precision in the redshift determination is compensated with the larger volume of the survey, the larger density of galaxies, and the possibility of analyzing different galaxy populations.

With large-volume surveys becoming the norm, it is timely to devise large-volume numerical simulations able to mimic, reproduce and control the various observational surveys. This paper aims at presenting two large cosmological $N$-body simulations, called Horizon Run 2 (HR2) and Horizon Run 3 (HR3), made using innovative computational facilities and forefront capabilities. Cosmological $N$-body simulations provide in fact a powerful tool to test the validity of cosmological models and galaxy formation mechanisms (Bertschinger 1998), as well as they represent a complementary and necessary benchmark to design and support the observational surveys. In particular, the advent of realistic cosmological $N$-body simulations, capable of reproducing successfully the global properties of the observed structure at large scales, has enabled significant progress in understanding the structure of dark matter halos, the DM clustering over a huge range of scales, the distribution of galaxies in space and time, the effects of the environment, the mass assembly history, and the nature of DM itself. For example, numerical studies have indicated that the hierarchical assembly of CDM halos yields approximately universal mass profiles (Navarro et al. 1996, 1997), strongly triaxial shapes with a slight preference for nearly prolate systems (i.e., Jing \& Suto 2002), presence of abundant but non-dominant substructure within the virialized region of a halo, and cuspy inner mass profiles (for instance see Springel et al. 2005). Numerical simulations are also crucial to DE studies, especially for characterizing BAO systematics (Crotts et al. 2005; Crocce \& Scoccimarro 2008; Kim et al. 2009). These are well controlled only with a detailed knowledge of all nonlinear effects associated, and can only be addressed by studying mock catalogs constructed from large $N$-body simulations. In this respect, comparison with $N$-body simulations is critical, as it allows one to calibrate the DE experiments with high accuracy.

While the current and future surveys of large-scale structure in the Universe demand larger and larger simulations, simulating large cosmological volumes with good mass resolution is clearly a major computational challenge. However, it is crucial to be able to reach volumes big enough to assess the statistical significance of extremely massive halos, almost always underrepresented in simulations that survey a small fraction of the Hubble volume. For example, till recently our understanding of the mass profile of massive haloes has 
been rather limited, derived largely from small numbers of individual realizations or from extrapolation of modes calibrated on different mass scales (Navarro et al. 1996, 1997; Moore et al. 1998; Klypin et al. 2001; Diemand et al. 2004; Reed et al. 2005). This is because, at high masses, enormous simulation volumes are required in order to collect statistically significant samples of these rare DM halos. Instead, individual halo simulations may result in biased concentration estimates in a variety of ways (Gao et al. 2008). At high masses we also know only approximately the redshift and mass dependence of halo concentration, even for the current concordance cosmology. Another example of how important is to simulate big volumes is related to DE science: larger volumes allow one to model more accurately the true power at large scales and the corresponding power spectrum, particularly in the case of the LRG distribution, which is critical to the BAO test (Kim et al. 2009). A large box size will guarantee small statistical errors in the power spectrum estimates: we need in fact to measure the acoustic peak scale down to an accuracy better than $1 \%$.

As explained in Neto et al. (2007) and in Gao et al. (2008), trying to combine different simulations of varying mass resolution and box size is instead potentially very risky, along with extrapolation techniques. To this end, Neto et al. (2007) brings the example of Macciò et al. (2007), who combined several simulations of varying mass resolution and box sizes in order to reach scales with $M \ll M_{*}$. Unfortunately, this approach comes with pitfalls because in order to resolve a statistically significant sample of haloes with masses of the order of $10^{10} h^{-1} \mathrm{M}_{\odot}$ one must use a considerably smaller simulation box (i.e., $14.2 h^{-1} \mathrm{Mpc}$ on a side); this implies a substantial lack of large-scale power, due to such small periodic realization, which may influence the result. The obvious way out is to increase the dynamic range of the simulation, so as to encompass a volume large enough to be representative, while at the same time having enough mass resolution to extend the analysis well above (or below) $M_{*}$.

In this view, the enormous volume of our HR2 and HR3, together with the large number of particles used, make our simulations ideal to characterize with minimal statistical uncertainty the dependence of the structural parameters of CDM halos on mass, formation time, departures from equilibrium, etc., and will be of great use for DE science and for all the upcoming photometric redshifts surveys.

The paper is organized as follows. In Section 2 we briefly discuss the standard numerical techniques in LSS analyses, and compare the number of particles and volumes of our simulations with other recent numerical studies. In Section 3, after a synthetic description of our previous Horizon Run 1 (HR1), we present our new simulations HR2 and HR3. In particular, we provide some technical details on the code used, the mass and force resolution, and the halo selection procedure. In Section 4 we introduce the 35 all-sky mock surveys along the past light cone ( 8 from the HR2 and 27 from the HR3), made in order to simulate the BOSS geometry. These mock catalogs are particularly suitable for DE studies (i.e., LRGs), and are already publicly available. We then present in Section 5 the first results from testing our new simulations; namely, the computation of the starting redshifts, mass functions, power spectra and two-point correlation functions. We conclude in Section 6, with a brief description on the importance and use of our simulations and catalogs. We are making the simulation data and mocks available to the community at http://astro.kias.re.kr/Horizon-Run23/.

\section{NUMERICAL METHODS}

Progressively sophisticated numerical techniques are used to carry out $N$-body simulations, with the goal of optimizing resources and performances by exploiting forefront technological developments.

Broadly speaking, there are two basic algorithms by which a simulation can be optimized: particle-mesh (PM) methods and tree methods - along with combinations of the two, such as the PM-Tree scheme. In the PM method, space is discretised on a mesh and particles are divided between the nearby vertices of the mesh, in order to compute the gravitational potential via Poisson's equation and fast Fourier transform (FFT) techniques. In the tree method, the volume is instead usually divided up into cubic cells and grouped so that only particles from nearby cells need to be treated individually, whereas particles in distant cells can be treated as a single large particle centered at its center of mass. Obviously, this latter scheme increases considerably the computational speed in particle-pair interactions.

Thanks to rapid advancements in memory and computing power capabilities, state-of-the-art simulations can be performed with billion of particles, since algorithmic and hardware development have increased the mass and spatial resolution by orders of magnitude. Hence, it is perhaps impressive to recall that one of the first $N$-body simulations run by Peebles (1970) utilized only 300 particles, while today we can simulate individual collapsed structures in a full cosmological context, and substructure halos can be resolved - with excellent approximations to CDM halos on a large mass and spatial range. To this end, Fig. 1 shows how our new HR2 and HR3 simulations compare with the previous HR1 and with other recent large-volume simulations, in terms of number of particles; for specific details on our simulations refer to the next section. Pale green filled hexagons in the figure represent the PM simulations made by our group, stars (pink) are the PM-Tree simulations carried out always by our group (see the various labels), while blue open circles are those performed by other authors. Note in particular the Millennium Run (Springel et al. 2005), with 10 billion particles and a box size of $500 h^{-1} \mathrm{Mpc}$, and the recent French collaboration $N$-body simulations (Teyssier et 


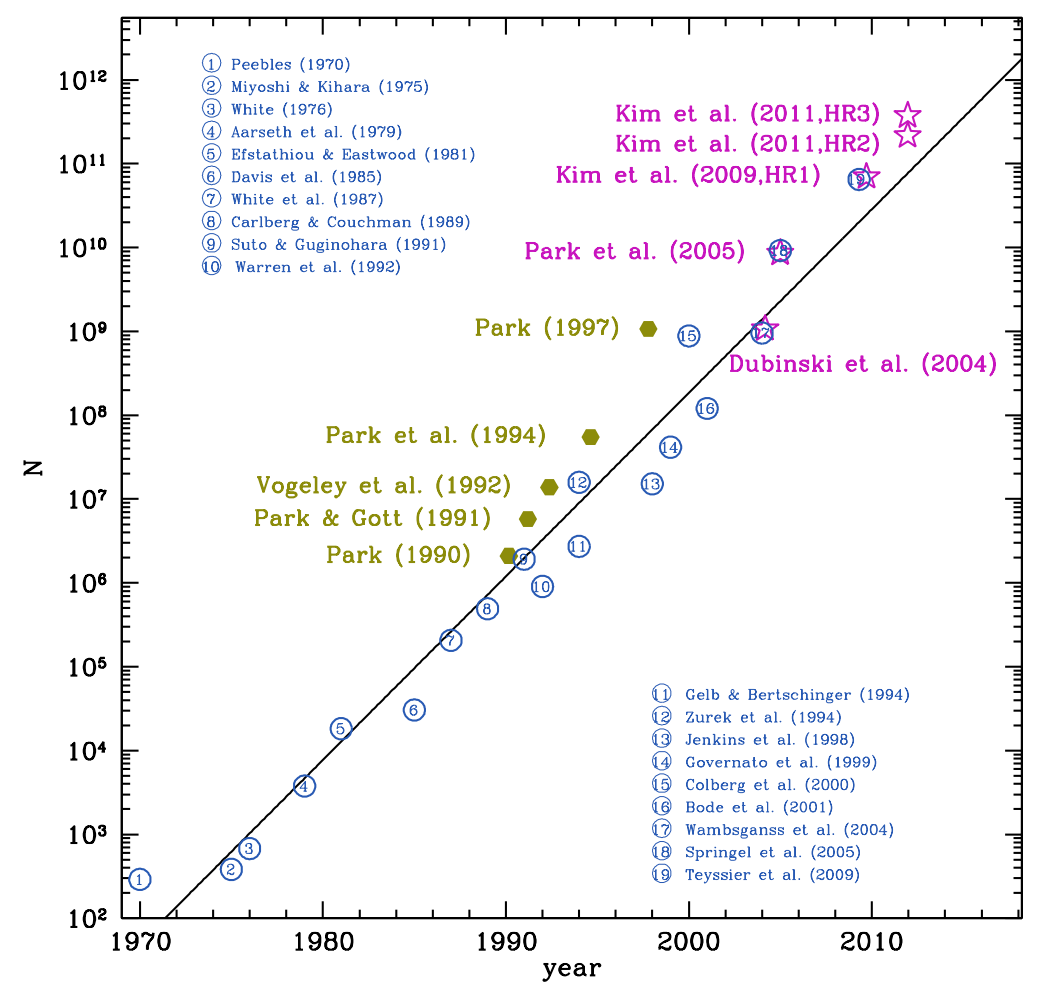

Fig. 1. - Evolution of the number of particles in $N$-body simulations versus time (years). Filled hexagons (pale green) are PM simulations made by our group; stars (pink) are PM-Tree simulations carried out by our group (the HR2 and HR3 are located in the upper-right corner); open circles (blue) are those performed by other authors - see the detailed legend in the figure. Note in particular the position of the Millennium Run (Springel et al. 2005; 10 billion particles, box size $500 h^{-1} \mathrm{Mpc}$ ), and the French collaboration (Teyssier et al. 2009; 68.7 billion particles, box size 2000h ${ }^{-1} \mathrm{Mpc}$ ). The solid line is the mean evolution of the simulation size.

al. 2009), made using 68.7 billion particles with a box size of $2000 h^{-1} \mathrm{Mpc}$. The solid line is the mean evolution of the simulation size taken from Springel et al. (2005), which is basically linear in logarithmic space, increasing a factor of 10 every 4.55 years.

The application of numerical methods in cosmology is in fact an exponentially growing field, in parallel with major technological developments; for a brief history of numerical studies see Diemand \& Moore (2009) and Kim et al. (2009). So far, efforts have been essentially focused both on investigating the formation of single halos with ultra-high resolution (Springel et al. 2008; Stadel et al. 2009), and on simulating structure formation in large boxes, to mimic the LSS in the Universe. Obviously, one needs to push numerical simulations to their limits, as the dynamical range of scales to be resolved is extremely large for addressing different types of cosmological problems: this study focuses on the latter part. What is interesting to notice here is that $N$-body simulations of the gravitational collapse of a collisionless system of particles actually pre-date the CDM model (see again Diemand \& Moore 2009). In effect, early simulations in the 1960's studied the formation of elliptical galaxies from the collapse of a cold top-hat perturbation of stars (van Albada 1961; Henon \& Heiles 1964; Peebles 1970). In the 70's, attempts were made to follow the expansion of a collapse of a spherical overdensity to relate to the observed properties of virialized structures such as galaxy clusters. In 1975, Groth \& Peebles made actual "cosmological" simulations using 1550 particles with $\Omega_{\mathrm{M}}=1$ and Poisson initial conditions (Groth \& Peebles 1975). After, Aarseth et al. (1979) used 4000 particles. Only in the 80 's, however, was it proposed that cosmic structure formation follows a dominant, non-baryonic CDM component, and later on the inflationary scenario in conjunction with CDM brought realistic initial conditions for $N$-body models. However, it was not until the simulations by Dubinski \& Carlberg that individual objects were simulated at sufficiently high resolution to resolve their inner structure on scales that could be compared with observations. Interestingly, Park (1990) was the first to use 4 million particles, a peak biasing scheme and a CDM, $\Omega_{\mathrm{M}} h=0.2$ model to simulate a 


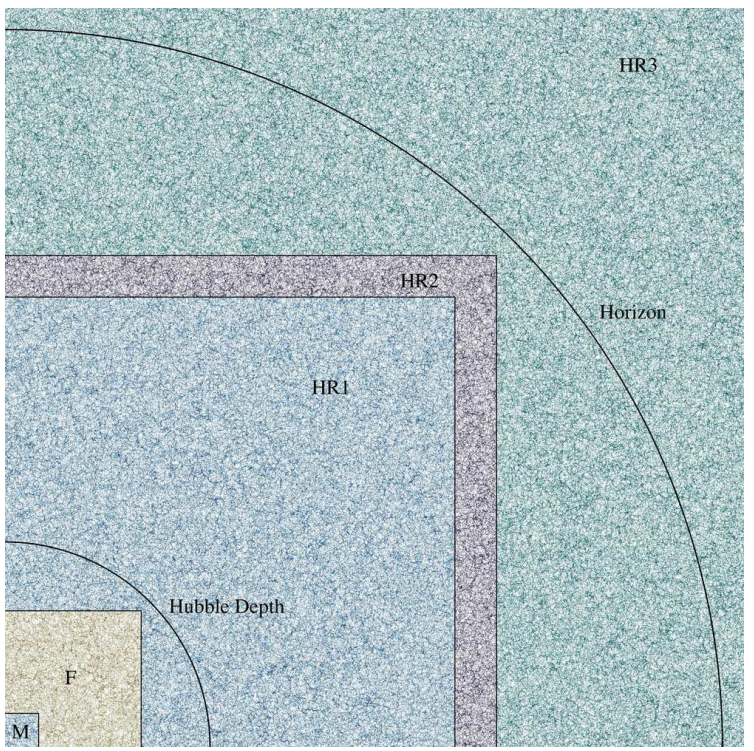

Fig. 2. - Visual comparison between the box sizes of different numerical simulations, via a $4.5 h^{-1} \mathrm{Mpc}$ thick slice through our HR3 $N$-body simulation at the present epoch. The box sizes are 500, 2000, 6595, 7200 and $10815 h^{-1} \mathrm{Mpc}$ for the Millennium Run, French collaboration, HR1, HR2 and HR3, respectively. The horizon and Hubble Depth (defined as $\mathrm{cH}_{0}^{-1}$ ) are also indicated in the figure, in comoving coordinates, to emphasize the various simulation volumes.

volume large enough to properly encompass the CfA Great Wall. After that, only in 2008 the first billion particle halo simulation Via Lactea II was published (Diemand et al. 2008), followed by Aquarius (Springel et al. 2008) and GHALO (Stadel et al. 2009), in a progressively increment of box size and number of particles till our HR1 (Kim et al. 2009) - which spanned the largest volume at the time.

To this end, Fig. 2 allows for a visual comparison on the massive increment in box size between our Horizon Runs with other recent cosmological $N$-body simulations. A $4.5 h^{-1} \mathrm{Mpc}$ slice through the HR3 is performed at the present epoch, in comoving coordinates. The box sizes are 500, 2000, 6595, 7200 and $10815 h^{-1} \mathrm{Mpc}$ for the Millennium Run, French collaboration, HR1, HR2 and HR3, respectively. The horizon and Hubble distance $\left(\mathrm{cH}_{0}^{-1}\right)$ are also indicated in the figure, to emphasize the various simulation volumes. Note the impressive improvement with respect to the Millennium Run box size.

\section{THE HORIZON RUNS}

After a brief description of our previous HR1 (Kim et al. 2009), in this section we present the main characteristics of the new HR2 and HR3, two of the largest cosmological $N$-body simulations to date. In particular, we provide detailed technical information regarding the code developed and used to perform the runs, the mass and force resolution, and the halo selection procedure. The main simulation parameters are summarized in Table 1 for convenience, and in the following parts we will refer to it - whenever necessary.

\subsection{The Horizon Run 1: Overview}

The HR1 (Kim et al. 2009) was the largest volume simulation ever run in 2009, with a box size of $6592 h^{-1} \mathrm{Mpc}$ on a side and $11418 h^{-1} \mathrm{Mpc}$ diagonally. The cosmological model adopted was a CDM concordance scenario with a cosmological constant (i.e., the LDCM model), having the basic parameters fixed by the WMAP 5-year data (Komatsu et al. 2009) listed in the second column of Table 1 . The initial linear power spectrum was calculated using the fitting function provided by Eisenstein \& Hu (1998). The initial conditions were generated on a $4120^{3}$ mesh with pixel size of $1.6 h^{-1} \mathrm{Mpc}$. It used a total of $4120^{3}=69.9$ billion CDM particles, representing the initial density field at $z_{\mathrm{i}}=23$. Those particles, initially perturbed from their uniform distribution, were gravitationally evolved by a TreePM code (Dubinski et al. 2004; Park et al. 2005) with force resolution of $160 h^{-1} \mathrm{kpc}$, for a total of 400 global time steps. The entire simulation-making process lasted 25 days on a TACHYON SUN Blade system (i.e., a Beowulf system with 188 nodes, each with $16 \mathrm{CPU}$ cores and 32 Gigabytes of memory), at the Korean KISTI Supercomputing Center. The simulation used 2.4 TBytes of memory, 20 TBytes of hard disk, and $1648 \mathrm{CPU}$ cores. The entire cube data were stored at various redshifts: $z=0,0.1,0.3,0.5,0.7,1$. In addition, the positions of the simulation particles were saved in a slice of constant thickness (equal to $64 h^{-1} \mathrm{Mpc}$ ), as they appeared in the past light cone. During the simulation, 8 equally-spaced (maximallyseparated) observers were located in the cube, in order to construct the mock SDSS-III LRG surveys; the positions and velocities of the particles were saved at $z<0.6$, as they crossed the past light cone. Subhalos were then found in the past light cone data, and used to simulate the SDSS-III LRG survey. For more details on how the corresponding mock catalogs were constructed see the next section, and Kim et al. (2009).

\subsection{The Horizon Runs 2 and 3: Improvements}

The major improvements of our new HR2 and HR3 with respect to the HR1 concern the number of particles used, the bigger box sizes (up to a factor of 4.4 in volume), and a considerably finer mass resolution. HR2 and HR3 have been made using $6000^{3}=216$ billions and $7210^{3}=374$ billion particles, spanning a volume of $\left(7.200 h^{-1} \mathrm{Gpc}\right)^{3}$ and $\left(10.815 h^{-1} \mathrm{Gpc}\right)^{3}$, respectively which range from 2600 to over 8800 times the volume of the Millennium Run. The mass resolution reaches down to $1.25 \times 10^{11} h^{-1} \mathrm{M}_{\odot}$, allowing to resolve galaxysize halos with mean particle separations of $1.2 h^{-1} \mathrm{Mpc}$ and $1.5 h^{-1} \mathrm{Mpc}$, respectively. Results span nearly six decades in mass. As with the HR1, these two new 


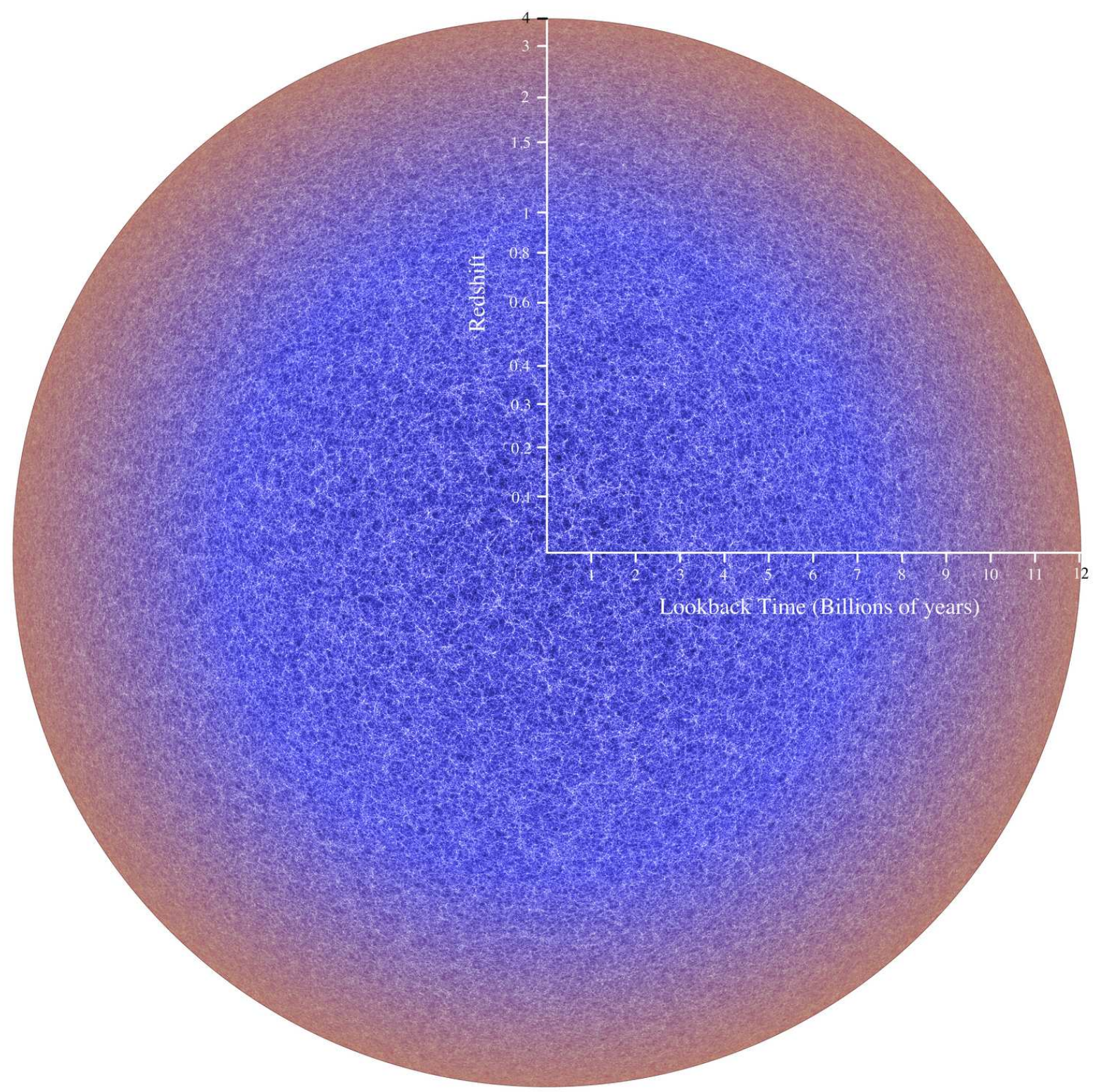

Fig. 3.- Example of the matter density in the past light cone space, obtained from a slice with $30 h^{-1} \mathrm{Mpc}$ thickness passing through the center of the HR3 simulation box. A mock observer is located at the center of the figure; the radius of the map corresponds to about $z=4$. The ticks along the horizontal axis indicate the lookback time, in units of billions of years, while the redshift is indicated along the vertical axis. The color scheme varies from blue to red as one goes to larger redshifts. The radial distance is linear in lookback time. The starting epoch of HR3 corresponds to a redshift of $z=27$.

simulations are based on the $\Lambda$ CDM cosmology, with parameters fixed by the WMAP 5-year data (Komatsu et al. 2009) listed in the third and fourth columns of Table 1. The initial redshifts of the simulations are $z_{i}=32$ and $z_{i}=27$, respectively, with 800 time steps for the HR2 and 600 for the HR3. For more details on the initial conditions, see Section 5.1. We also improved on the 'old fashion' linear power spectrum by adopting the CAMB source (http://camb.info/sources), which provides a better measurement of the $\mathrm{BAO}$ scale: this is essential for a more accurate determination of the cosmological parameters using the BAO constraint.

The positions of the simulation particles were saved in a slice of constant thickness (equal to $30 h^{-1} \mathrm{Mpc}$ ), as they appeared in the past light cone. To construct the various mock catalogs, eight observers were evenly located in the simulation box of the HR2, and twenty seven in the box of the HR3. Each observer volume covers a space spanning from $z=0$ to $z=0.7$, without overlaps: this means that the survey volumes are 


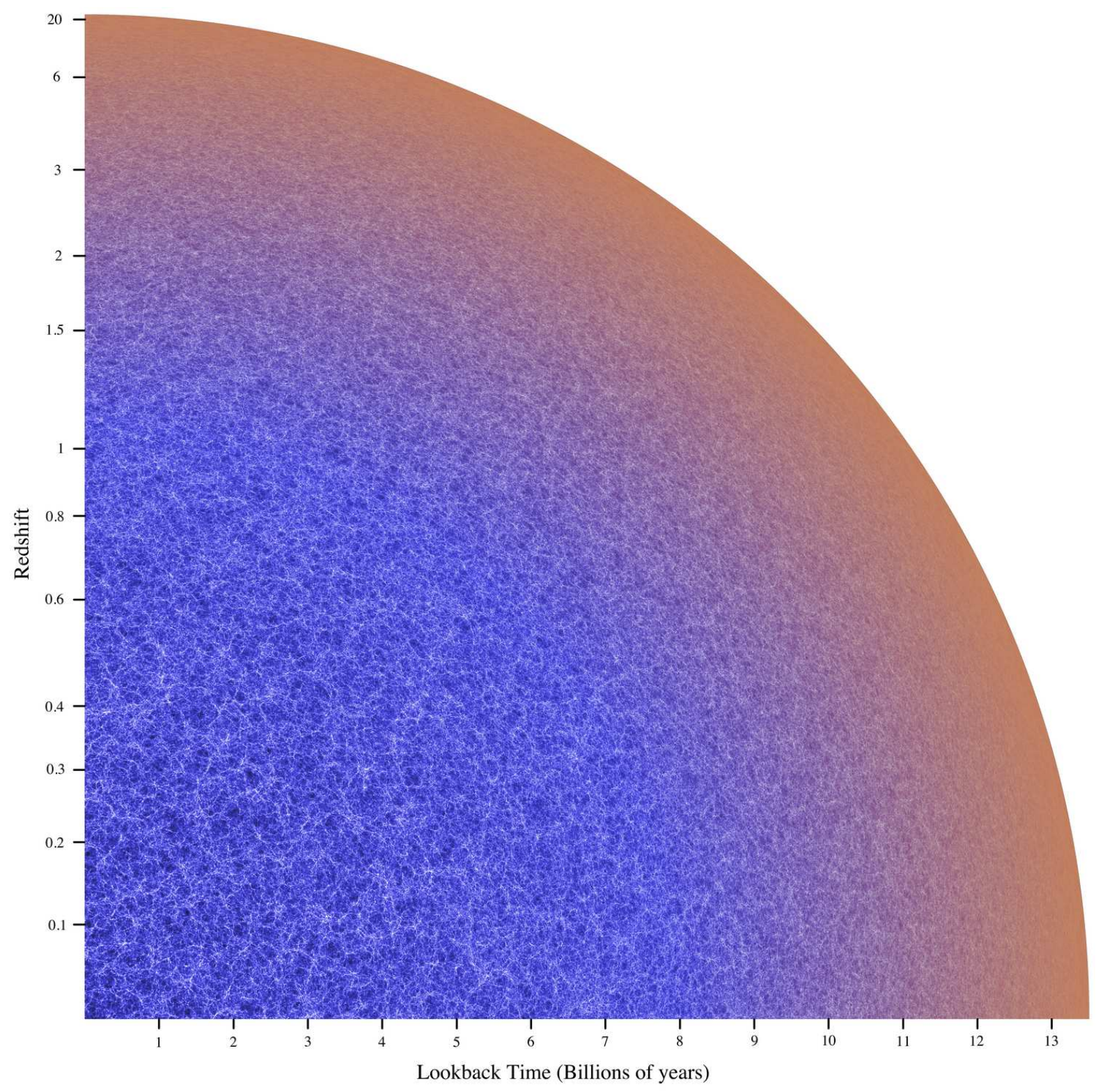

Fig. 4. - In false colors, a quarter-wedge slice density map in the past light cone is shown, from the HR3. Color scheme as in the previous figure. The width is $30 h^{-1} \mathrm{Mpc}$, and the wedge reaches a redshift of $z=27$. The density is measured using the spline kernel (a technique widely used in SPH), with 5 nearest particles. The ticks along the horizontal axis indicate the lookback time, in units of billions of years, while the redshift is indicated along the vertical axis. Note that the starting epoch of the HR3 corresponds to the maximum redshift of $z=27$.

totally independent. In addition, there is one big mock survey region for each simulation. This volume reaches $z=1.85$ (HR2) and $z=4$ (HR3), respectively, and the center is located at the origin of the simulation box. Note in particular that the latter mock survey covers the range out to $z=2.5$, where BOSS data allow one to measure the 3D BAO using Lyman- $\alpha$ clouds seen in absorption in front of many quasar lines of sights.

Fig. 3 is an example of the matter density in the past light cone space from the HR3 (the width is $\left.30 h^{-1} \mathrm{Mpc}\right)$. A mock observer is located at the center of the figure; the radius of the map corresponds to about $z=4$. The ticks along the horizontal axis indicate the lookback time, in units of billions of years, while the redshift is indicated along the vertical axis. The color scheme varies from blue to red as one goes to larger redshifts. Note that the starting epoch of the HR3 corresponds to the maximum redshift of $z=27$. The distribution of the CDM particles is converted to a density field using the variable-size spline kernel con- 


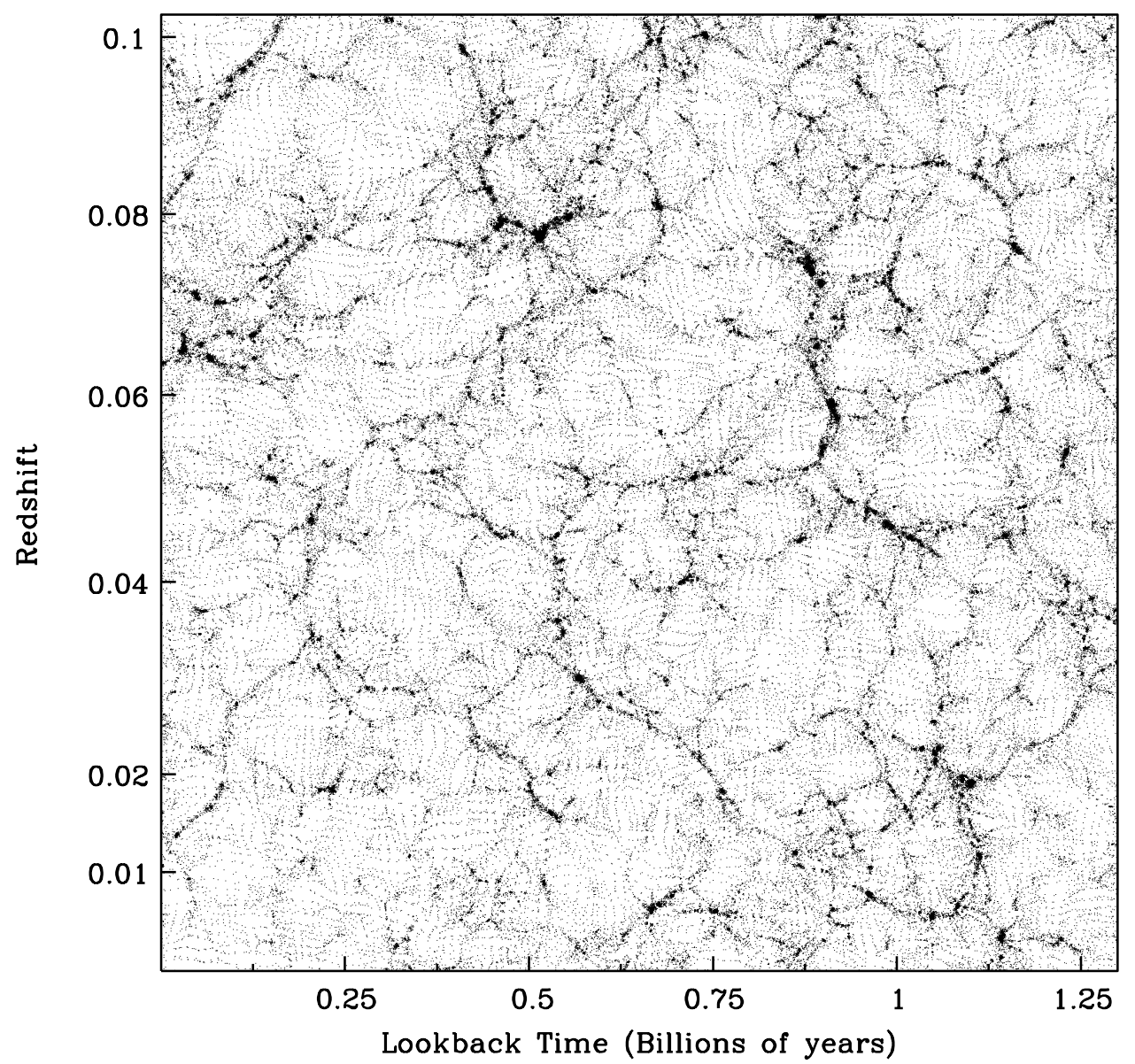

Fig. 5. - Particle distribution from a small region (i.e., out to $z \simeq 0.1$ ) of the entire mock survey volume shown in Fig. 4, which reveals the typical cosmic web-like pattern: individual halos, filaments and sheets can be easily seen, connected in a network, with voids encompassing the volume in between. Particles in the survey volume are shown if they are inside the slice volume with width $\Delta z$ corresponding to $7.5 h^{-1} \mathrm{Mpc}$. The plot demonstrates the large dynamical range of our simulation, capable of resolving the clustering at small scales.

taining 5 CDM particles - a technique well-known in smoothed-particle hydrodynamics (SPH).

Fig. 4 is another example obtained from our HR3. In false colors, a quarter-wedge slice density map in the past light cone is shown. The width is $30 h^{-1} \mathrm{Mpc}$, and the wedge reaches $z=27$. The color scheme varies as in the previous figure. The ticks along the horizontal axis indicate the lookback time, in units of billions of years, while the redshift is indicated along the vertical axis; the starting epoch of the HR3 is $z=27$.

Fig. 5 shows the particle distribution from a small region (i.e., out to $z \simeq 0.1$ ) of the entire mock survey volume, which clearly reveals the typical cosmic weblike pattern: one can easily distinguish individual halos, filaments and sheets, connected in a network, with voids encompassing the volume in between. The plot demonstrates the large dynamical range of our simulations, which are able to resolve correctly individual structures at small scales.

\subsection{Improving the GOTPM Code}

The Grid-of-Oct-Trees-Particle-Mesh code, called GOTPM, is a parallel, cosmological $N$-body code based on a hybrid scheme using the PM and Barnes-Hut octtree algorithm, originally devised by Dubinski et al. (2004). We used an improved version of this code for our HR2 and HR3 simulations. Specifically, we implemented a new procedure which allows us to describe more accurately the particle positions. In fact, when dealing with billions of particles, single precision (adopted in the original version of GOTPM) is inaccurate for representing their positions. This is because 
Table 1.

Detailed specifics of our Horizon Run $N$-body simulations.

\begin{tabular}{cccc}
\hline \hline & HR1 & HR2 & HR3 \\
\hline Model & WMAP5 & WMAP5 & WMAP5 \\
$\Omega_{\mathrm{M}}$ & 0.26 & 0.26 & 0.26 \\
$\Omega_{\mathrm{b}}$ & 0.044 & 0.044 & 0.044 \\
$\Omega_{\Lambda}$ & 0.74 & 0.74 & 0.74 \\
$H_{0}\left[100 \mathrm{~km} \mathrm{~s}{ }^{-1} \mathrm{Mpc}^{-1}\right]$ & 0.96 & 0.96 & 0.96 \\
$\sigma_{8}$ & 72 & 72 & 72 \\
Box size $\left[h^{-1} \mathrm{Mpc}\right]$ & 0.794 & 0.794 & 0.794 \\
No. of grids for initial conditions & 6592 & 7200 & 10815 \\
No. of CDM particles & $4120^{3}$ & $6000^{3}$ & $7210^{3}$ \\
Starting redshift & $4120^{3}$ & $6000^{3}$ & $7210^{3}$ \\
No. of global time steps & 23 & 32 & 27 \\
Mean particle separation $\left[h^{-1} \mathrm{Mpc}\right]$ & 400 & 800 & 600 \\
Particle mass $\left[10^{11} h^{-1} \mathrm{M}_{\odot}\right]$ & 1.6 & 1.2 & 1.5 \\
Minimum halo mass $\left(30\right.$ particles) $\left[10^{11} h^{-1} \mathrm{M}_{\odot}\right]$ & 8.96 & 1.25 & 2.44 \\
Mean separation of minimum mass PSB halos $\left[h^{-1} \mathrm{Mpc}\right]$ & 13.08 & 37.5 & 73.2 \\
\hline
\end{tabular}

the significant bits in single precision are only 24 , which allow for about 7 significant digit numbers. The resulting position error in single precision is about $N / 2^{24}$, with $N$ the number of particles in one dimension. For example, the particles in the $7210^{3}$ simulation have a maximum intrinsic positioning error of $0.04 \%$, which becomes significant when their clustering is high and when those particles are packed in small areas. In these situations, using single precision provides highly inaccurate results. A possible (and obvious) solution is to use double precision instead; however, this would require too much memory space (i.e., an additional $30 \%$ increment). To solve the problem, we devised a simpler method which does not require any additional space but only some more calculation time. In essence, instead of determining the positions of the particles using the position vector in the GOTPM code, we used the corresponding offset vector (or displacement vector), from the pre-initial Lagrangian position. We then calculated the position of the particle directly from its index and particle offset vector. By adopting this procedure, the positioning error is about $d_{(x, y, z)} / 2^{24}$, where $d_{(x, y, z)}$ is the displacement from the Lagrangian position. This value is significantly smaller than $N$.

In addition, in the GOTPM each particle requires a total of 40 bytes; four of them are used to save the position and velocity, eight are for the particle index, and other eight are allocated for a pointer which is used to build a linked list in the Tree mode. Since the particle pointer is not used in the PM mode, this memory space is recycled for the density mesh and for the FFT workspace.

\subsection{Halo Selection}

Identifying and deciding which material belongs to a halo and what lies beyond it is clearly a non-trivial question. This is because DM halos are dynamical structures, constantly accreeting material and often substantially out of virial equilibrium. In these circumstances, halos evolve quickly so that the parameters used to specify their properties change rapidly and thus are ill-defined. Furthermore, in the case of an ongoing major merger even the definition of the halo center becomes ambiguous.

In the Horizon Runs, halos are first identified via a standard Friend-of-Friend (FoF) procedure. Then subhalos are found (out of FoF halos) with a subhalo finding technique, developed by Kim \& Park (2006) and Kim et al. (2008). This method allows one to identify physically self-bound (PSB) dark matter subhalos not tidally disrupted by larger structures at the desired epoch. This procedure does not discard any information on the subhalos which is actually in the $N$-body simulations, and would be thrown away in a Halo Occupation Distribution (HOD) analysis using just simple FoF halos. Note in fact that the FoF algorithm is a percolation scheme that makes no assumptions about halo geometry, but may spuriously group distinct halos together into the same object. In particular, we have applied the parallel version of the FoF halo finding method to identify virialized structures from the simulation particles. Linked particles with mutual distance less than $0.2 \times d_{\text {mean }}$, with $d_{\text {mean }}$ the mean particle separation, are grouped. In addition, subhalos in a FoF halo are found by a new version of the PSB method, more advanced than the previous one (Kim \& Park 
2006) in that it adopts adaptive density fields rather than the rectangular grid density fields. Densities at the particle positions are measured using the SPH density allocation scheme, and each particle is linked to 30 nearest neighbors. The neighbor links are used for building hierarchical isodensity contours around local density peaks. These coordinate-free density allocation and neighbor search are much more effective for identifying subhalos in crowded regions.

\section{MOCK SURVEYS}

In this section, first we briefly describe our previous mock catalogs made from the HR1, in support of the SDSS-III. We then introduce our new 35 all-sky mock surveys along the past light cones, made from the HR2 and HR3 to simulate the BOSS geometry and already publicly available at http://astro.kias.re.kr/HorizonRun23/.

Regardless of the specific simulation considered, the following general procedure is used to construct a mock catalog. For each simulation time step, we track particles which are located in the light cone shell surrounded by two spherical surfaces of radii $\left(d-\Delta_{1 / 2}, d+\Delta_{1 / 2}\right)$ (i.e., inner and outer shells), with $d$ being the comoving distance from the observer at the given time step, and $\Delta$ the difference between the comoving distance at the next step (subscript 1) or at the previous step (subscript 2) - with respect to that at the current step. We then calculate the particle distances and flag them if they are in the shell. The radius and width of the shell (and so the volume) are calculated according to the cosmological model and the simulation time and step size. Obviously, the particles can cross the shell boundary between different time steps, since they are moving in space. If that happens, they can be double detected in adjacent time steps - or totally missed. In the former case, if a particle is double detected between consecutive time steps, we average its position and velocity. For the latter case, in order not to miss a particle, we create a buffer zone with constant width $\left(\Delta_{\mathrm{R}}=0.1\right.$ of the simulation pixel), and make two regions in the inner and outer shell boundaries, respectively; we then identify particles inside the buffer zone. If a particle is detected in both regions of the buffer zone, we average its position and velocity and save the information in the light cone particle list. This method has been devised to account for double detections, and in order not to miss particles - which happens frequently due to the finite size of the simulation time step.

\subsection{Mocks from the HR1: Overview}

To make mock SDSS-III LRG surveys from the HR1, we placed observers at 8 different locations in the simulation cube, and carried out all-sky surveys up to $z=0.6$. These are all past light cone data. The effects of our choices of the starting redshift, time step and force resolution were discussed in detail in Kim et al.
(2009). In particular, we used the Zel'dovich redshifts and the FoF halo multiplicity function to estimate their effects (see also the next section).

During the simulation we located 8 equally-spaced (maximally-separated) observers in the simulation cube, and saved the positions and velocities of the particles at $z<0.6$ as they cross the past light cone. We assumed that the SDSS-III survey would produce a volume-limited LRG sample with constant number density of $3 \times 10^{-4}\left(h^{-1} \mathrm{Mpc}\right)^{-3}$. In our simulation we varied the minimum mass limit of subhalos to match the number density of selected subhalos (the mock LRGs) with this number at each redshift. For example, the mass limit yielding the LRG number density of $3 \times 10^{-4}\left(h^{-1} \mathrm{Mpc}\right)^{-3}$ was found to be $1.33 \times 10^{13} h^{-1} \mathrm{M}_{\odot}$ and $9.75 \times 10^{12} h^{-1} \mathrm{M}_{\odot}$ at $z=0$ and $z=0.6$, respectively. We then checked how well the mock LRG sample reproduced the physical properties of the existing LRG sample - see for this Kim et al. (2008), Gott et al. (2008), Gott et al. (2009), Choi et al. (2010). We found a good match from 1 to $140 h^{-1} \mathrm{Mpc}$, particularly in the case of the shape of the correlation function. In addition, the BAO scale and the LSS topology could be very accurately calibrated with these mocks.

\subsection{New Mocks from the HR2 and HR3}

To simulate the ongoing BOSS survey and construct the various mock catalogs, we evenly positioned observers at the centers of eight (HR2) and twenty seven (HR3) sub-cubes of the entire simulation boxes. Each observer volume covers a space spanned from $z=0$ to $z=0.7$, without overlaps: this means that the survey volumes are totally independent. In addition, we considered one mock observer covering the entire simulation region, with survey depth down to $z=1.85$ for the HR2, and $z=4$ for the HR3. For each simulation time step, we track particles which are located in the light cone shell surrounded by two spherical surfaces as explained before, and account for double detections and potentially missing particles.

In particular, our mocks can be used to simulate the distribution of LRGs in the BOSS survey. This is done as follows: first, obtain FoF halos with standard linking length of $0.2 \times d_{\text {mean }}$ from the mock particle distributions. Then identify subhalos in each FoF halo using the PSB method (Kim et al. 2008), and call them PSB halos. Kim, Park, \& Choi (2008) showed that there is a strong one-to-one relation between PSB halos and galaxies, under the condition that a more massive PSB halo contains a brighter galaxy. Hence, apply this relation to the PSB halo list to simulate the distributions of LRGs - note that their mean observed number density is expected to be $\simeq 3 \times 10^{-4}\left(h^{-1} \mathrm{Mpc}\right)^{-3}$. It is then possible to find the minimum mass of PSB halos which can have LRGs with luminosity higher than the BOSS observation limit, by using their expected number density. In Fig. 6 we show the minimum PSB halo mass as a function of redshift, for the HR2 and HR3. 


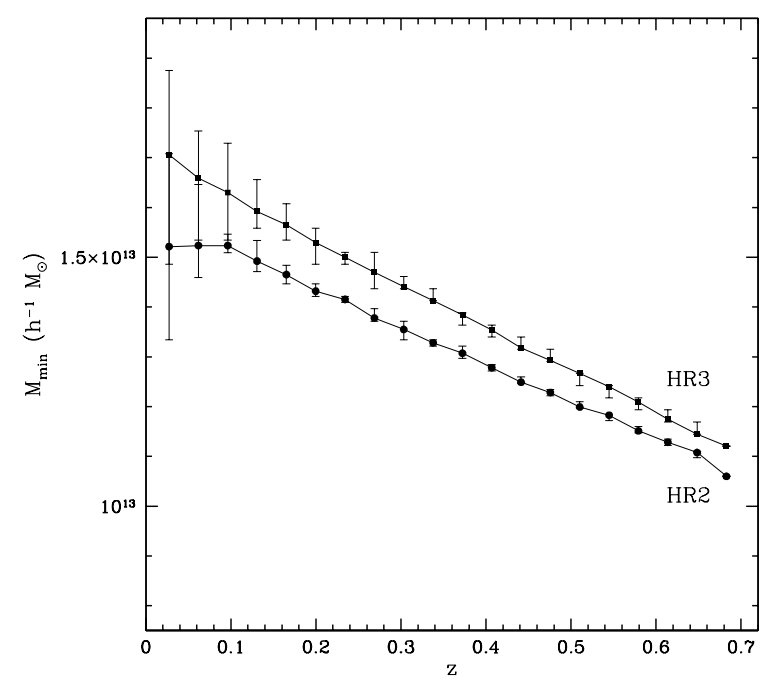

Fig. 6.- Minimum PSB halo mass as a function of redshift, for the HR2 and HR3. This plot is useful for constructing mock catalogs which simulate the distribution of LRGs in the BOSS survey. See the main text for more details.

Note that the two trends are similar, independently of the simulation considered, but their amplitudes differ substantially. This is mainly due to the different resolutions of the simulations. Clearly, a finer resolution allows us to detect subhalos which are not seen at lower resolutions. Consequently, massive subhalos can be divided into several less massive subhalos at higher resolutions - the well-known cloud-in-cloud problem. This is also why in Fig. 6 the HR2 results have lower amplitude, since the HR2 has the highest mass resolution with respect to the HR3. Therefore, for a given mass scale, the HR3 has more subhalos than HR2 and a larger minimum PSB halo mass than HR2.

\section{FIRST RESULTS}

In this section we present the first results from the analysis of the HR2 and HR3 simulations. In particular, we have measured power spectra, correlation functions, mass functions and basic halo properties with percent level accuracy, and verified that they correctly reproduce the $\Lambda \mathrm{CDM}$ theoretical expectations, in excellent agreement with linear perturbation theory. In what follows, we also provide some more details on how the initial conditions have been fixed. The results described here are only basic checks, as the main goal of this study is to present our new simulations; in the upcoming papers we will provide more detailed scientific analyses, with a particular focus on topology, BAO, and galaxy formation science.

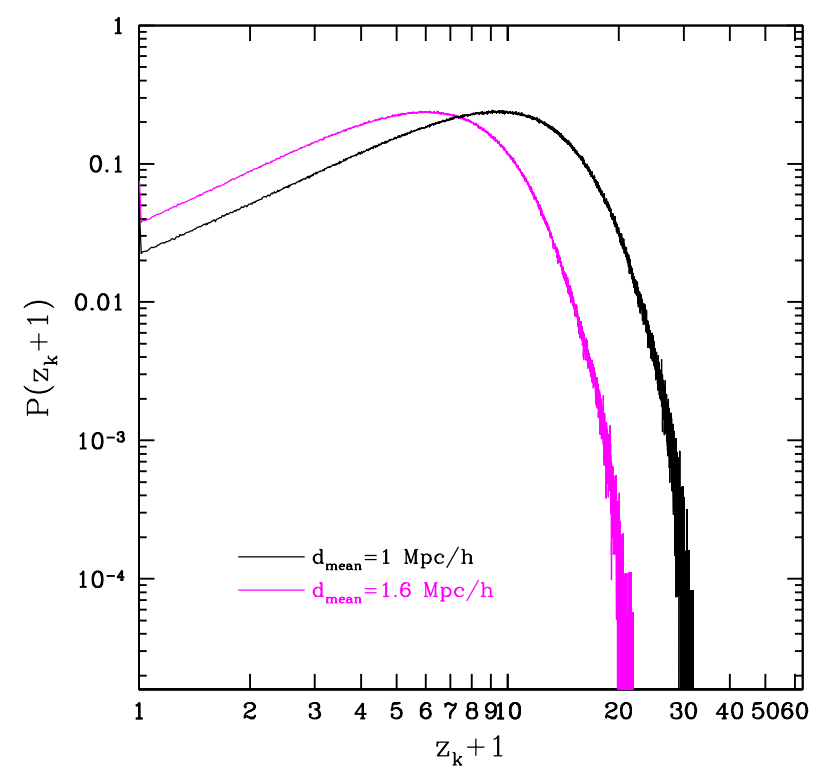

Fig. 7.- Distributions of the Zel'dovich redshifts for two different pixel sizes (see the main text for definitions). The black curve corresponds to $d_{\text {mean }}=1 h^{-1} \mathrm{Mpc}$, while for the purple one $d_{\text {mean }}=1.5 h^{-1} \mathrm{Mpc}$. This figure is used to determine the starting redshifts of the HR2 and HR3 simulations.

\subsection{Initial Conditions: Starting Redshift and Time Steps}

We used a TreePM code (Dubinski et al. 2004) to generate the initial Zel'dovich displacements for our HR2 and HR3, which adopts the first-order Lagrangian perturbation scheme. Correctly determining the proper initial conditions is essential for $N$-body simulations. In fact, as Crocce et al. (2006) pointed out, improper initial conditions may cause errors in the resulting halo mass function and bias function for massive halos. Those errors originate by starting the simulations at too low redshift, while using first-order techniques for the initial particle displacements and velocities. Obviously, a way out is to use second order perturbation theory to correct for these effects. Alternatively, one can simply adopt the first-order Lagrangian perturbation scheme and choose the starting redshifts of the simulations so that the Lagrangian shifts of the particles are not larger than the pixel spacing: this correspond to $z_{\mathrm{i}}=32$ for the $\mathrm{HR} 2$, and $z_{\mathrm{i}}=27$ for the HR3 (see the discussion below). In fact, if the initial displacement is larger than the pixel spacing, a particle may not fully experience the local differential gravitational potential, and consequently the shift calculated using the first-order scheme could not fully reflect the fluctuations at the pixel scale. Fig. 7 shows how we quantitatively estimate the proper starting redshifts for our initial conditions. As in Kim et al. (2009), we introduce the Zel'dovich redshift $z_{\mathrm{k}}$ of the particles, defined 
as the epoch when its Zel'dovich displacement becomes equal to the pixel size - either in the $x, y$ or $z$ directions. Two simulations are considered, tagged by the mean particle separation $d_{\text {mean }}$, or equivalently by the pixel size. In the first one $d_{\text {mean }}=1 h^{-1} \mathrm{Mpc}-$ a value close to that of the HR2, which has a mean separation of $1.2 h^{-1} \mathrm{Mpc}$; in the second one $d_{\text {mean }}=1.6 h^{-1} \mathrm{Mpc}$, and is used for the HR3, whose mean separation is $1.5 h^{-1} \mathrm{Mpc}$. To measure the distributions, we perform an initial setting moving $256^{3}$ particles from the initial conditions defined on a $256^{3}$-size mesh. Each distribution shows a power-law increase with $z_{\mathrm{k}}$ and a sharp drop after a peak. It can be clearly seen that no particle experiences a shift larger than the pixel size at $z=32$ when $d_{\text {mean }}=1 h^{-1} \mathrm{Mpc}$ (black curve, at the HR2 resolution), and at $z=27$ when $d_{\text {mean }}=1.5 h^{-1} \mathrm{Mpc}$ (purple curve, at the HR3 resolution). This justifies our safe choice of the initial redshifts for the HR2 and HR3 simulations.

We adopted 800 time steps to evolve the CDM particles from $z=32$ to 0 for the HR2, 600 time steps from $z=27$ to 0 for the HR3, and set the force resolution to $0.1 \times d_{\text {mean }}$. The choice of the various time steps is motivated next.

\subsection{Mass Functions}

Because of the relatively large step size and the lower force resolution, the question arises as to whether or not the HR2 and HR3 simulations have sufficient time and force resolution to correctly model the formation of halos. To this end, we can simply evaluate if the simulations retain sufficient power to resolve small structures by comparing the FoF halo multiplicity functions computed directly from the HR2 and HR3 with numerical fitting formulae obtained from high-resolution simulations. This is particularly true for the population of less massive halos: in fact, the coarse time step size or a poor force resolution may destroy smaller structures more easily. By computing the halo multiplicity functions, Fig. 8 shows that indeed our large-volume simulations are able to resolve correctly about six decades in mass. Recall that the multiplicity function, a quantity independent of redshift, is the differential distribution function of the normalized fluctuation amplitude of dark halos for each mass element, defined as

$$
f[\sigma(M)]=n(M)\left|\frac{\mathrm{d} M}{\mathrm{~d} \ln \sigma}\right| \frac{M}{\bar{\rho}}
$$

where $n(M)$ is the number density of halos with mass $M, \sigma(M)$ is the standard deviation of the density field smoothed with a tophat of mass scale $M$, and $\bar{\rho}$ is the background density.

In the figure, the HR2 results are displayed in purple, those of the HR3 in grey. Filled circles are measurements performed at $z=0$, open circles correspond to a redshift $z=0.14$ for the HR2 and to $z=0.32$ for the HR3, and filled triangles are at $z=0.32$ for the HR2

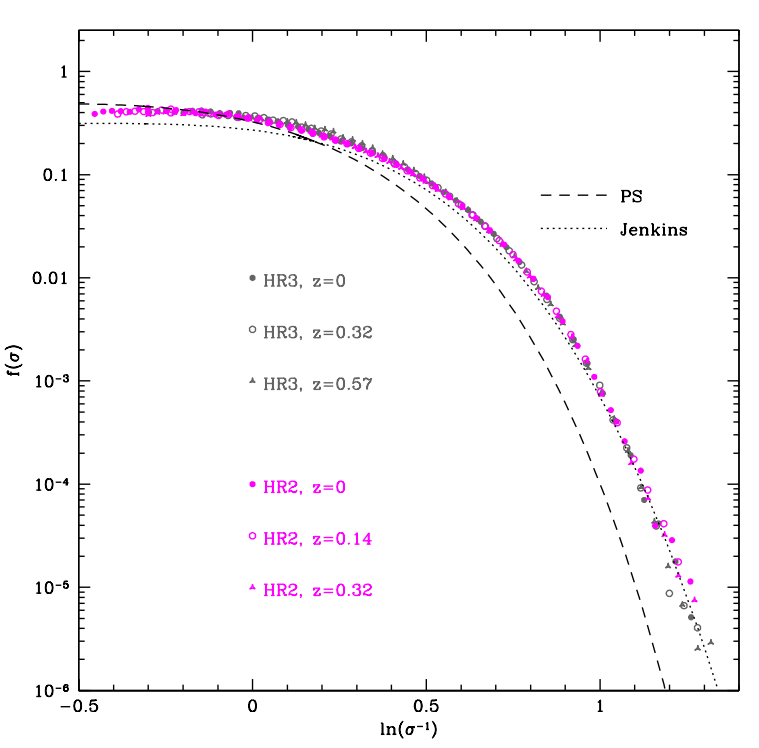

Fig. 8.- Halo multiplicity function computed from the HR2 and HR3. A standard FoF identification scheme is adopted. The HR2 results are displayed in purple, those of the HR3 in grey. Filled circles are measurements performed at $z=0$, open circles correspond to a redshift $z=0.14$ for the HR2 and to $z=0.32$ for the HR3, and filled triangles are at $z=0.32$ for the HR2 and at $z=0.57$ for the HR3. The minimum halo mass which can be resolved in the HR2 (30 simulation particles) corresponds to $M_{30} \simeq 3.75 \times 10^{12} h^{-1} \mathrm{M}_{\odot}$, while $M_{30} \simeq 7.32 \times 10^{12} h^{-1} \mathrm{M}_{\odot}$ for the HR3. The dashed line shows the Press \& Schechter (1974) mass function (PS); the dotted line is instead the fit provided by Jenkins et al. (2001). See the main text for more details.

and at $z=0.57$ for the HR3. In particular, the minimum halo mass which can be resolved in the HR2 (30 simulation particles are used) corresponds to $M_{30} \simeq$ $3.75 \times 10^{12} h^{-1} \mathrm{M}_{\odot}$, while $M_{30} \simeq 7.32 \times 10^{12} h^{-1} \mathrm{M}_{\odot}$ for the HR3. In the plot, the dashed line shows the Press \& Schechter (1974) mass function (PS); the dotted line is instead the fit provided by Jenkins et al. (2001). From the figure it can be seen that the Jenkins et al. (2001) curve provides a good fit to our measurements, with only minor deviations (but see Lukic et al. 2007 for more details on this scatter).

Our measurements at different redshifts are all consistent. Hence, we safely conclude that the FoF halo multiplicity function is complete down to the simulation mass resolution for halos: the simulations correctly model the formation of halos, and are not affected by our choices of the force resolution and the number of time steps.

\subsection{Power Spectra and Correlation Functions}

Next, we compute the power spectrum and twopoint correlation function for each individual large- 


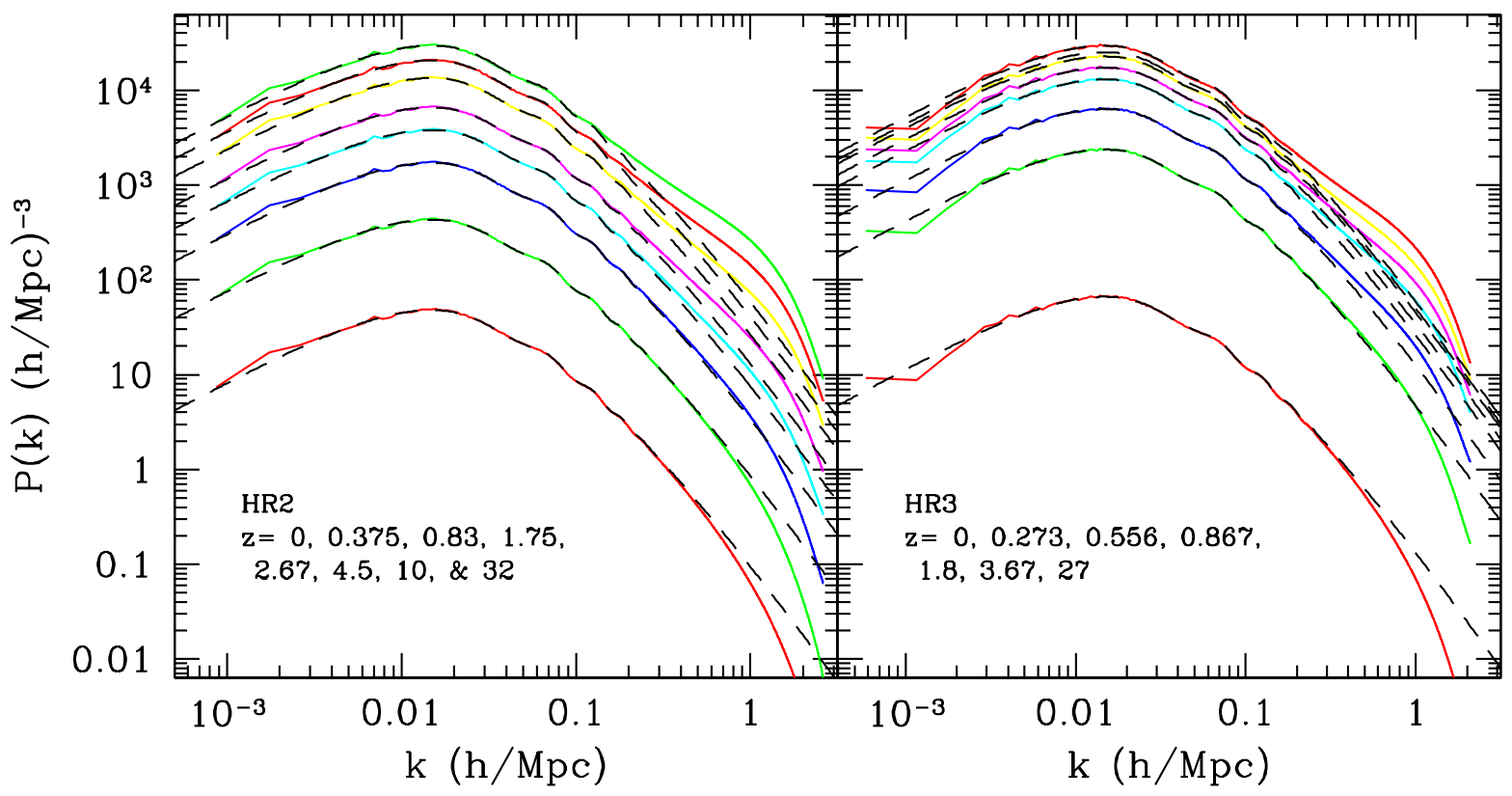

Fig. 9. - Power spectra of the CDM density field at various redshifts in comoving coordinates, as measured from the HR2 (left panel) and HR3 (right panel). The lower lines in the figure are the initial conditions, and the top ones are at $z=0$. Solid lines are measurements from simulations; long-dashed lines are obtained by linearly evolving the (linear) power spectra to the corresponding redshifts.

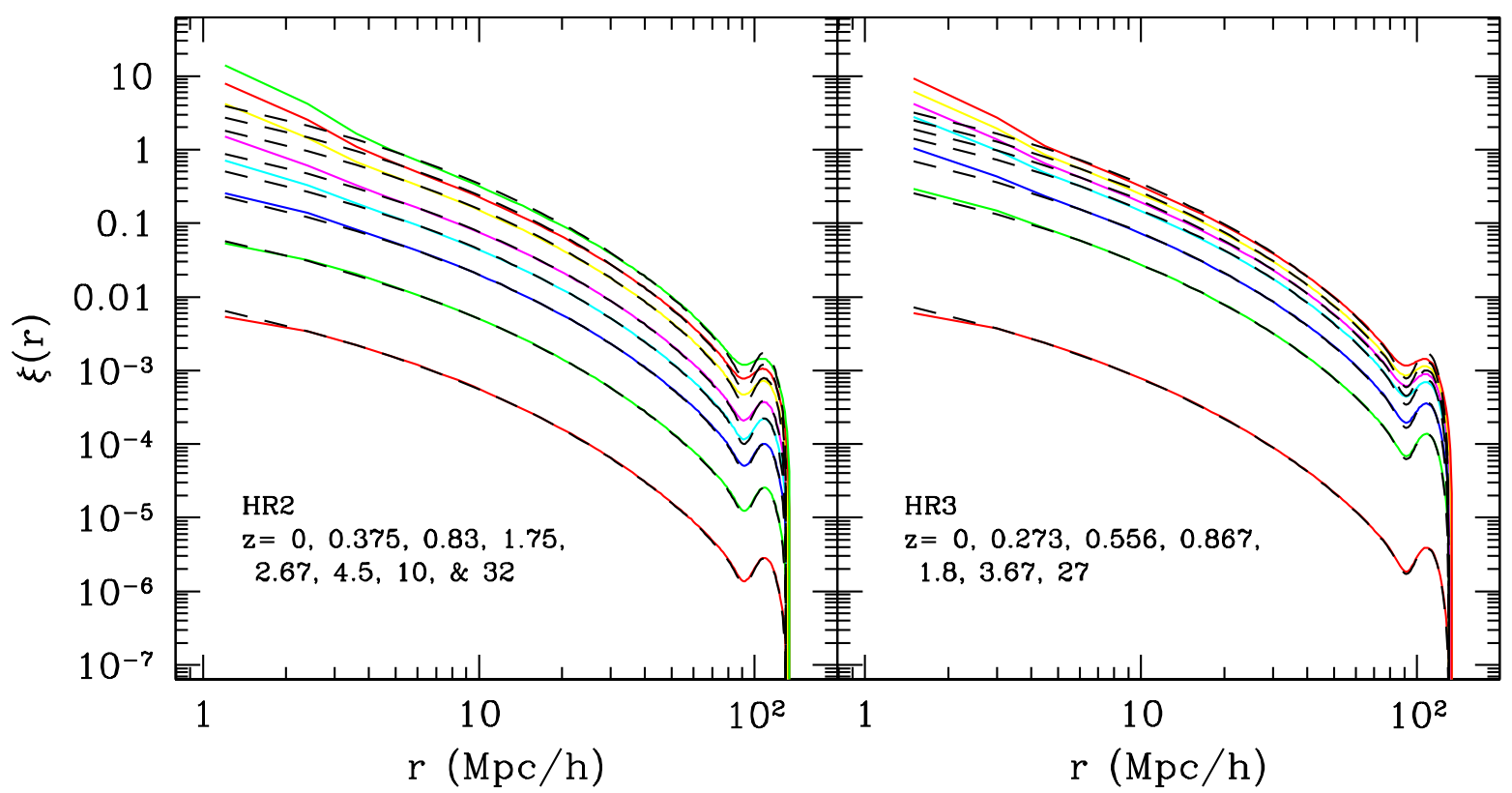

Fig. 10. - Two-point correlation functions of the CDM density field at various redshifts in comoving coordinates, as measured from the HR2 (left panel) and HR3 (right panel). As in the previous figure, the lower lines are initial conditions and the top ones are at $z=0$. Solid lines are again measurements from simulations; long-dashed lines are obtained by Fourier transforming the linearly evolved (linear) power spectra to the corresponding redshifts. Note the BAO bump, at different redshift intervals.

volume simulation. In particular, Fig. 9 shows the power spectra of the CDM density field at various redshifts in comoving coordinates, as measured from the HR2 (left panel) and HR3 (right panel). Solid 
lines in the figure are simulation results, while longdashed lines are obtained by linearly evolving the (linear) power spectra to the corresponding redshifts. Note that the redshift increases from the bottom to the top of the panels; therefore the lower curves correspond to the initial conditions (i.e., $z=32$ for the HR2, and $z=27$ for the HR3), while the top ones are at $z=0$.

Fig. 10 shows the corresponding evolution of the two-point correlation function of the matter density field (in comoving coordinates), for the same redshift intervals considered in the previous figure. Again, the left panel highlights results from the HR2, while the right one presents measurements from the HR3. Solid lines are simulation results, long-dashed lines are obtained by Fourier transforming the linearly evolved (linear) power spectra to the corresponding redshifts. Note that the lower curves correspond to the initial conditions, while the top ones are at $z=0$. The BAO bumps at different redshifts are clearly visible; in a dedicated forthcoming paper, we will present a detailed study on the statistical significance of the BAO feature in the HR2 and HR3. Note the tiny amount of noise because we are sampling such a large volume, which allows us to reduce considerably the uncertainty in the BAO bump position. In addition, the systematics on the BAO position due to nonlinear effects and biasing are small, and can be corrected for with high accuracy.

\section{CONCLUSIONS}

The main goal of this paper was to present in detail two new large cosmological $N$-body simulations, HR2 and HR3, made using $6000^{3}=216$ billions and $7210^{3}=$ 374 billion particles, and spanning a volume of $(7.200$ $\left.h^{-1} \mathrm{Gpc}\right)^{3}$ and $\left(10.815 h^{-1} \mathrm{Gpc}\right)^{3}$, respectively. The volumes of these two simulations are considerably bigger than our previous HR1 volume, up to a factor of 4.4 , and the mass resolution has been improved (down to $\left.1.25 \times 10^{11} h^{-1} \mathrm{M}_{\odot}\right)$; this allows to resolve large-galaxy (i.e., LRG galaxy) sized halos, with mean particle separations of $1.2 h^{-1} \mathrm{Mpc}$ (HR2) and $1.5 h^{-1} \mathrm{Mpc}$ (HR3). The main characteristics of the simulations have been described in detail in Section 3, including the code developed and used to perform the runs, the mass and force resolution, and the halo selection procedure. The basic simulation parameters are listed in Table 1.

Planned next generation photometric redshift galaxy surveys such as BOSS (Schlegel et al. 2009), BigBOSS (Schlegel et al. 2011), DES (Abbott et al. 2005), LSST (Tyson et al. 2004), WFIRST (Green et al. 2011) and Euclid (Cimatti et al. 2008) will span volumes considerably larger than the current data sets, providing a dramatic improvement in the accuracy of the constraints on cosmological parameters; to this end, our simulations will be of great help for these surveys.

In particular, in support of the SDSS-III, we made a total of 35 all-sky mock catalogs along the past light cone up to $z=0.7,8$ from the HR2 and 27 from the HR3, to simulate the BOSS geometry (Section 4). The simulations and mock surveys are already publicly available to the community, and they should be useful for a variety of studies in cosmology and astrophysics, ranging from large-scale structure topology, baryon acoustic oscillations, dark energy and the characterization of the expansion history of the Universe, till galaxy formation science.

In Section 5 we also presented the first results from the analysis of the HR2 and HR3 simulations. In particular, we have measured power spectra, correlation functions, mass functions and basic halo properties with percent level accuracy, and verified that they correctly reproduce the $\Lambda \mathrm{CDM}$ theoretical expectations, in excellent agreement with linear perturbation theory. For example, by computing the halo multiplicity functions we proved that our large-volume simulations are able to resolve correctly about six decades in mass. Much more scientific analyses can be carried out with these simulations, and in the upcoming papers we will focus in particular on topology, BAO, and galaxy formation science.

Clearly, one may question the utility of performing such large-volume, expensive and computationally challenging simulations. Why not running instead many smaller simulations, with different initial conditions? Several reasons were already pointed out in the introductory part; essentially, the main goal in doing so is to cover large scale modes accurately, which is crucial for dark energy and BAO science (i.e., systematic effects), for the statistical study of rare massive halos, and for the formation of unusually large scale structures. In fact, as we argued at the beginning, extremely massive halos are almost always underrepresented in simulations that survey a small fraction of the Hubble volume, and in order to reach a statistically significant sample of these objects one needs simulations with enormous dynamic range. By contrast, combining different simulations of varying mass resolution is instead potentially very risky (i.e., Neto et al. 2007; Gao et al. 2008).

While we strive to carry out simulations in as large volumes as possible, we also need high resolutions to sample accurately the underlying gravitational potential wells. The HR2 and HR3 achieve both criteria: their volumes are unprecedentedly large, but their mass resolutions allow us to resolve large-galaxy-sized (LRG) halos; therefore, they are ideal to characterize with minimal statistical uncertainty the dependence of the key structural parameters of CDM halos on mass, spin, formation time, etc., and will be of great use for DE science (i.e., BOSS) and for all the upcoming photometric redshifts surveys.

In particular, a topical issue which can be addressed with our simulations is the nature of DE and the determination of the DE EoS parameter $w(z)$ as a function of redshift. A standard technique, which has received much attention lately, is the measurement of the 
BAO scale using LRGs (Eisenstein et al. 2005). In this case, being able to control the systematics is imperative, and can only be achieved via large volume $N$ body simulations such as the HR2 and HR3. Note in fact that the typical box sizes usually adopted for these studies in previous literature range from $500 h^{-1} \mathrm{Mpc}$ to $2000 h^{-1} \mathrm{Mpc}$, but those boxes are inadequate because they are too close to the baryon oscillation scale - which is of the order of $108 h^{-1} \mathrm{Mpc}$. Instead, it is essential to be able to model the power spectrum accurately at large scales, and to have a large box size so that the statistical errors in the power spectrum are small.

The HR2 and HR3 also permit us to assess the statistical significance of unusually large structures such as the Sloan Great Wall which, with a length of 1.37 billion light years (Gott et al. 2005), is considerably more massive than predicted by previous $N$-body simulations (i.e., Springel et al. 2005). The large number of halos in our simulations allows us to study in detail deviations from the mean trends and the possible presence of such systems with unusually high concentrations of galaxy haloes, or unusually low densities. We will present a detailed study focused on this issue in a separate publication. We will also investigate several other applications, which involve the large scale topology in relation to dark energy and distance measurements, the detailed modeling of the integrated SachsWolf effect (Yoo, Fitzpatrick, \& Zaldarriaga 2009) and the comparison with counts of LRGs in the sky, and even applications of first order corrections to our simulations in order to capture general relativity effects.

Our simulations and mock surveys are already publicly available at http://astro.kias.re.kr/Horizon-Run23/. Please cite this paper whenever you use the simulation data.

\section{ACKNOWLEDGMENTS}

This work was supported by the Supercomputing Center/Korea Institute of Science and Technology Information with supercomputing resources, including technical support (KSC-2011-G2-02). We thank the Korea Institute for Advanced Study for providing computing resources (KIAS Center for Advanced Computation Linux Cluster System) for this work.

\section{REFERENCES}

Aarseth, S. J., Turner, E. L., \& Gott, J. R. 1979, NBody Simulations of Galaxy Clustering. I - Initial Conditions and Galaxy Collapse Times, ApJ, 228, 664

Abbott, T., et al. 2005, The Dark Energy Survey, astro$\mathrm{ph} / 0510346$

Albrecht, A., et al. 2006, Report of the Dark Energy Task Force, astro-ph/0609591

Bertschinger, E. 1998, Simulations of Structure Formation in the Universe, ARAA, 36, 599
Bett, P., Eke, V., Frenk, C. S., Jenkins, A., Helly, J., \& Navarro, J. 2007, The Spin and Shape of Dark Matter Haloes in the Millennium Simulation of a $\Lambda$ Cold Dark Matter Universe, MNRAS, 376, 215

Blake, C., et al. 2008, The Wiggle Z Dark Energy Survey, Astronomy \& Geophysics, 49, 19

Bode, P., Bahcall, N. A., Ford, E. B., \& Ostriker, J. P. 2001, Evolution of the Cluster Mass Function: GPC3 Dark Matter Simulations, ApJ, 551, 15

Carlberg, R. G., \& Couchman, H. M. P. 1989, Mergers and Bias in a Cold Dark Matter Cosmology, ApJ, 340,47

Carnero, A., Sanchez, E., Crocce, M., Cabre, A., \& Gaztanaga, E. 2011, Clustering of Photometric Luminous Red Galaxies - II. Cosmological Implications from the Baryon Acoustic Scale, arXiv:1104.5426

Choi, Y.-Y., Park, C., Kim, J., Gott, J. R., Weinberg, D. H., Vogeley, M. S., \& Kim, S. S. 2010, Galaxy Clustering Topology in the Sloan Digital Sky Survey Main Galaxy Sample: A Test for Galaxy Formation Models, ApJS, 190, 181

Cimatti, A., et al. 2008, GMASS Ultradeep Spectroscopy of Galaxies at $\mathrm{z} \sim 2$. II. Superdense Passive Galaxies: How Did They Form and Evolve?, A\&A, 482, 21

Colberg, J. M., White, S. D. M., Yoshida, N., et al. 2000, Clustering of Galaxy Clusters in Cold Dark Matter Universes, MNRAS, 319, 209

Cole, S., et al. 2005, The 2dF Galaxy Redshift Survey: Power-Spectrum Analysis of the Final Data Set and Cosmological Implications, MNRAS, 362, 505

Colless, M., et al. 2001, The 2dF Galaxy Redshift Survey: Spectra and Redshifts, MNRAS, 328, 1039

Crocce, M., \& Scoccimarro, R. 2008, Nonlinear Evolution of Baryon Acoustic Oscillations, Phys. Rev. D., 77,3533

Crocce, M., Pueblas, S., \& Scoccimarro, R. 2006, Transients from Initial Conditions in Cosmological Simulations, MNRAS, 373, 369

Crotts, A., et al. 2005, Joint Efficient Dark-energy Investigation (JEDI): a Candidate Implementation of the NASA-DOE Joint Dark Energy Mission (JDEM), astro-ph/0507043

Dalal, N., Dore, O., Huterer, D., \& Shirokov, A. 2008, Imprints of Primordial Non-Gaussianities on Large-Scale Structure: Scale-Dependent Bias and Abundance of Virialized Objects, Phys. Rev. D., 77, 123514

Davis, M., Efstathiou, G., Frenk, C. S., \& White, S. D. M. 1985, The Evolution of Large-Scale Structure in a Universe Dominated by Cold Dark Matter, ApJ, 292, 371

Desjacques, V., \& Seljak, U. 2010, Signature of Primordial Non-Gaussianity of $\phi^{3}$ Type in the Mass Function and Bias of Dark Mtter Haloes, Phys. Rev. D., 81,3006 
Desjacques, V., Seljak, U., \& Iliev, I. T. 2009, ScaleDependent Bias Induced by Local Non-Gaussianity: a Comparison to N-Body Simulations, MNRAS, 396, 85

Diemand, J., \& Moore, B. 2009, The Structure and Evolution of Cold Dark Matter Halos, arXiv:0906.4340

Diemand, J., Kuhlen, M., Madau, P., Zemp, M., Moore, B., Potter, D., \& Stadel, J. 2008, Clumps and Streams in the Local Dark Matter Distribution, Nature, 454, 735

Diemand, J., Moore, B., \& Stadel, J. 2004, Convergence and Scatter of Cluster Density Profiles, MNRAS, 353, 624

Dubinski, J., Kim, J., Park, C., \& Humble, R. 2004, GOTPM: a Parallel Hybrid Particle-Mesh Treecode, New Astronomy, 9, 111

Efstathiou, G., \& Eastwood, J. W. 1981, On the Clustering of Particles in an Expanding Universe, MNRAS, 194, 503

Eisenstein, D. J., et al. 2005, Detection of the Baryon Acoustic Peak in the Large-Scale Correlation Function of SDSS Luminous Red Galaxies, ApJ, 633, 560

Eisenstein, D. J., \& Hu, W. 1999, Power Spectra for Cold Dark Matter and Its Variants, ApJ, 511, 5

Eisenstein, D. J., \& Hu, W. 1998, Baryonic Features in the Matter Transfer Function, ApJ, 496, 605

Gao, L., et al. 2008, The Redshift Dependence of the Structure of Massive Cold Dark Matter Haloes, MNRAS, 387, 536

Gao, L., \& White, S. D. M. 2007, Assembly Bias in the Clustering of Dark Matter Haloes, MNRAS, 377, 5

Gaztanaga, E., Cabre, A., \& Hui, L. 2009, Clustering of Luminous Red Galaxies - IV. Baryon Acoustic Peak in the Line-of-Sight Direction and a Direct Measurement of $H(z)$, MNRAS, 399, 1663

Gelb, J. M., \& Bertschinger, E. 1994, Cold Dark Matter. 1: The Formation of Dark Halos, ApJ, 436, 467

Gott, J. R., Choi, Y.-Y., Park, C., \& Kim, J. 2009, Three-Dimensional Genus Topology of Luminous Red Galaxies, ApJ, 695, 45

Gott, J. R., et al. 2008, Genus Topology of Structure in the Sloan Digital Sky Survey: Model Testing, ApJ, 675,16

Gott, J. R., Juric, M., Schlegel, D., Hoyle, F., Vogeley, M., Tegmark, M., Bahcall, N., \& Brinkmann, J. 2005, A Map of the Universe, ApJ, 624, 463

Gott, J. R., Dickinson, M., \& Melott, A. L. 1986, The Sponge-Like Topology of Large-Scale Structure in the Universe, ApJ, 306, 341

Governato, F., Babul, A., Quinn, T., et al. 1999, Properties of Galaxy Clusters: Mass and Correlation Functions, MNRAS, 307, 949
Green, J., et al. 2011, Wide-Field InfraRed Survey Telescope (WFIRST) Interim Report, arXiv: 1108.1374

Groth, E. J., \& Peebles, P. J. E. 1975, N-Body Studies of the Clustering of Galaxies, BAAS, 7, 425

Henon, M., \& Heiles, C. 1964, The Applicability of the Third Integral of Motion: Some Numerical Experiments, AJ, 69, 73

Hill, G. J., Gebhardt, K., Komatsu, E., \& MacQueen, P. J. 2004, The Hobby-Eberly Telescope Dark Energy Experiment, AIPC, 743, 224

Jee, I., Park, C., \& Kim, J. 2011, A Second-Order Bias Model for the Logarithmic Halo Mass Density, ApJ, submitted

Jenkins, A., et al. 2001, The Mass Function of Dark Matter Haloes, MNRAS, 321, 372

Jenkins, A., Frenk, C. S., Pearce, F. R., et al. 1998, Evolution of Structure in Cold Dark Matter Universes, ApJ, 499, 20

Jeong, D., \& Komatsu, E. 2009, Primordial NonGaussianity, Scale-dependent Bias, and the Bispectrum of Galaxies, ApJ, 703, 1230

Jing, Y. P., Suto, Y., \& Mo, H. J. 2007, The Dependence of Dark Halo Clustering on Formation Epoch and Concentration Parameter, ApJ, 657, 664

Jing, Y. P., \& Suto, Y. 2002, Triaxial Modeling of Halo Density Profiles with High-Resolution N-Body Simulations, ApJ, 574, 538

Kaiser, N., et al. 2002, Pan-STARRS: A Large Synoptic Survey Telescope Array, Proc. SPIE, 4836, 154

Kazin, E. A., Blanton, M. R., Scoccimarro, R., McBride, C. K., \& Berlind, A. A. 2010, The Baryonic Acoustic Feature and Large-Scale Clustering in the Sloan Digital Sky Survey Luminous Red Galaxy Sample, ApJ, 710, 1444

Kim, J., Park, C., Gott, J. R., \& Dubinski, J. 2009, The Horizon Run N-Body Simulation: Baryon Acoustic Oscillations and Topology of Large-scale Structure of the Universe, ApJ, 701, 1547

Kim, J., Park, C., \& Choi, Y.-Y. 2008, A SubhaloGalaxy Correspondence Model of Galaxy Biasing, ApJ, 683, 123

Kim, J., \& Park, C. 2006, A New Halo-Finding Method for N-Body Simulations, ApJ, 639, 600

Klypin, A., Kravtsov, A. V., Bullock, J. S., \& Primack, J. R. 2001, Resolving the Structure of Cold Dark Matter Halos, ApJ, 554, 903

Komatsu, E., et al. 2011, Seven-year Wilkinson Microwave Anisotropy Probe (WMAP) Observations: Cosmological Interpretation, ApJS, 192, 18

Komatsu, E., et al. 2009, Five-Year Wilkinson Microwave Anisotropy Probe Observations: Cosmological Interpretation, ApJS, 180, 330 
Kowalski, M., et al. 2008, Improved Cosmological Constraints from New, Old, and Combined Supernova Data Sets, ApJ, 686, 749

Li, Y., Mo, H. J., \& Gao, L. 2008, On Halo Formation Times and Assembly Bias, MNRAS, 389, 1419

LoVerde, M., Hui, L., \& Gaztanaga, E. 2008, Lensing Corrections to Features in the Angular Two-Point Correlation Function and Power Spectrum, Phys. Rev. D., 77, 3512

Lukic, J., Heitmann, K., Habib, S., Bashinsky, S., \& Ricker, P. M. 2007, The Halo Mass Function: HighRedshift Evolution and Universality, ApJ, 671, 1160

Macciò, A. V., Dutton, A. A., van den Bosch, F. C., Moore, B., Potter, D., \& Stadel, J. 2007, Concentration, Spin and Shape of Dark Matter Haloes: Scatter and the Dependence on Mass and Environment, MNRAS, 378, 55

Matsubara, T. 2004, Correlation Function in Deep Redshift Space as a Cosmological Probe, ApJ, 615, 573

Miyoshi, K., \& Kihara, T. 1975, Development of the Correlation of Galaxies in an Expanding Universe, PASJ, 27, 333

Montesano, F., Sanchez, A. G., \& Phleps, S. 2010, A New Model for the Full Shape of the Large-Scale Power Spectrum, MNRAS, 408, 2397

Moore, B., Governato, F., Quinn, T., Stadel, J., \& Lake, G. 1998, Resolving the Structure of Cold Dark Matter Halos, ApJ, 499, 5

Navarro, J. F., Frenk, C. S., \& White, S. D. M. 1997, A Universal Density Profile from Hierarchical Clustering, ApJ, 490, 493

Navarro, J. F., Frenk, C. S., \& White, S. D. M. 1996, The Structure of Cold Dark Matter Halos, ApJ, 462, 563

Neto, A., et al. 2007, The Statistics of $\Lambda$ CDM Halo Concentrations, MNRAS, 381, 1450

Park, C. 1997, A Particle-Mesh Code for the Next Generation Cosmological N-Body Simulations, JKAS, 30, 191

Park, C. 1990, Large N-Body Simulations of a Universe Dominated by Cold Dark Matter MNRAS, 242, 59

Park C., \& Kim, Y. R. 2010, Large-Scale Structure of the Universe as a Cosmic Standard Ruler, ApJL, $715, \mathrm{~L} 185$

Park, C., Kim, J., \& Gott, J. R. 2005, Effects of Gravitational Evolution, Biasing, and Redshift Space Distortion on Topology, ApJ, 633, 1

Park, C., Colley, W. N., Gott, J. R., Ratra, B., Spergel, D. N., \& Sugiyama, N. 1998, Cosmic Microwave Background Anisotropy Correlation Function and Topology from Simulated Maps for MAP, ApJ, 506, 473
Park, C., Vogeley, M. S., Geller, M. J., \& Huchra, J. P. 1994, Power Spectrum, Correlation Function, and Tests for Luminosity Bias in the CfA Redshift Survey, ApJ, 431, 569

Park, C., \& Gott, J. R. 1991, Simulation of Deep Oneand Two-Dimensional Redshift Surveys, MNRAS, 249, 288

Peebles, P. J. E. 1982, The Peculiar Velocity around a Hole in the Galaxy Distribution, ApJ, 257, 438

Peebles, P. J. E. 1970, Structure of the Coma Cluster of Galaxies, ApJ, 75, 13

Percival, W. J., et al. 2010, Baryon Acoustic Oscillations in the Sloan Digital Sky Survey Data Release 7 galaxy Sample, MNRAS, 401, 2148

Percival, W. J., Cole, S., Eisenstein, D. J., Nichol, R. C., Peacock, J. A., Pope, A. C., \& Szalay, A. S. 2007, Measuring the Baryon Acoustic Oscillation scale using the Sloan Digital Sky Survey and 2dF Galaxy Redshift Survey, MNRAS, 381, 1053

Perlmutter, S., et al. 1999, Measurements of Omega and Lambda from 42 High-Redshift Supernovae, ApJ, 517, 565

Press, W. H., \& Schechter, P. 1974, Formation of Galaxies and Clusters of Galaxies by Self-Similar Gravitational Condensation, ApJ, 187, 425

Reed, D., et al. 2005, Evolution of the Density Profiles of Dark Matter Haloes, MNRAS, 357, 82

Reid, B. A., et al. 2010, Cosmological Constraints from the Clustering of the Sloan Digital Sky Survey DR7 Luminous Red Galaxies, MNRAS, 404, 60

Riess, A. G., et al. 1998, Observational Evidence from Supernovae for an Accelerating Universe and a Cosmological Constant, AJ, 116, 1009

Sanchez, A. G., Crocce, M., Cabre, A., Baugh, C. M., \& Gaztanaga, E. 2009, Cosmological Parameter Constraints from SDSS Luminous Red Galaxies: a New Treatment of Large-Scale Clustering, MNRAS, 400,1643

Sanchez, A. G., et al. 2006, Cosmological Parameters from Cosmic Microwave Background Measurements and the Final 2dF Galaxy Redshift Survey Power Spectrum, MNRAS, 366, 189

Schlegel, D., et al. 2011, The BigBOSS Experiment, arXiv: 1106.1706

Schlegel, D., White, M., \& Eisenstein, D. 2009, The Baryon Oscillation Spectroscopic Survey: Precision measurement of the absolute cosmic distance scale, The Astronomy and Astrophysics Decadal Survey, Science White Papers, 314

Shandarin, S., Habib, S., \& Heitmann, K. 2010, Origin of the Cosmic Network in $\Lambda$ CDM: Nature vs Nurture, Phys. Rev. D., 81, 3006 
Spergel, D. N., et al. 2003, First-Year Wilkinson Microwave Anisotropy Probe (WMAP) Observations: Determination of Cosmological Parameters, ApJS, 148,175

Springel, V., et al. 2008, The Aquarius Project: the Subhaloes of Galactic Haloes, MNRAS, 391, 1685

Springel, V., et al. 2005, Simulations of the Formation, Evolution and Clustering of Galaxies and Quasars, Nature, 435, 629

Stadel, J., et al. 2009, Quantifying the Heart of Darkness with GHALO - a Multibillion Particle Simulation of a Galactic Halo, MNRAS, 398, 21

Sugiyama, N. 1995, Cosmic Background Anisotropies in Cold Dark Matter Cosmology, ApJS, 100, 281

Suto, Y., \& Suginohara, T. 1991, Redshift-Space Correlation Functions in the Cold Dark Matter Scenario, ApJL, 370, L15

Teyssier, R., et al. 2009, Full-Sky Weak-Lensing Simulation with 70 Billion Particles, A\&A, 497, 335

Tyson, J. A., \& LSST 2004, The Large Synoptic Survey Telescope Science Requirements, AAS, 20510801

van Albada, G. B. 1961, Evolution of Clusters of Galaxies under Gravitational Forces, AJ, 66, 590

Verde, L., \& Matarrese, S. 2009, Detectability of the Effect of Inflationary Non-Gaussianity on Halo Bias, ApJ, 706, 91

Vogeley, M. S., Park, C., Geller, M. J., \& Huchra, J. P. 1992, Large-Scale Clustering of Galaxies in the CfA Redshift Survey, ApJ, 391, 5

Wambsganss, J., Bode, P., \& Ostriker, J. P. 2004, Giant Arc Statistics in Concord with a Concordance Lambda Cold Dark Matter Universe, ApJL, 606, L93

Warren, M. S., Quinn, P. J., Salmon, J. K., \& Zurek, W. H. 1992, Dark Halos Formed via Dissipationless Collapse. I - Shapes and Alignment of Angular Momentum, ApJ, 399, 405

White, S. D. M. 1976, The Dynamics of Rich Clusters of Galaxies, MNRAS, 177, 717

White, S. D. M., Davis, M., Efstathiou, G., \& Frenk, C. S. 1987, Galaxy Distribution in a Cold Dark Matter Universe, Nature, 330, 451

White, S. D. M., \& Rees, M. J. 1978, Core Condensation in Heavy Halos - A Two-Stage Theory for Galaxy Formation and Clustering, MNRAS, 183, 341

Yoo, J., Fitzpatrick, A. L., \& Zaldarriaga, M. 2009, New Perspective on Galaxy Clustering as a Cosmological Probe: General Relativistic Effects, Phys. Rev. D., 80, 3514

York, D. G., et al. 2000, The Sloan Digital Sky Survey: Technical Summary, AJ, 120, 1579
Zheng, Z., \& Weinberg, D. H. 2007, Breaking the Degeneracies between Cosmology and Galaxy Bias, ApJ, 659, 1

Zurek, W. H., Quinn, P. J., Salmon, J. K., \& Warren, M. S. 1994, Large-Scale Structure after COBE: Peculiar Velocities and Correlations of Cold Dark Matter Halos, ApJ, 431, 559 